\title{
Evidence of sperm entry into assumed unfertilized human oocytes after sub-zonal sperm microinjection
}

\section{F.Urner, P.G.Bianchi, A.Campana and D.Sakkas ${ }^{1}$}

Clinique de Stérilité et $d^{`}$ Endocrinologie Gynécologique, Hôpital Cantonal Universitaire de Genève, 32 Bd de la Cluse,

1211 Genève 14 , Switzerland

${ }^{1}$ To whom correspondence should be addressed

Sub-zonal sperm microinjection (SUZI) as a treatment for male factor infertility can facilitate fertilization, however, in many cases oocytes remain unfertilized even though the sperm is placed in close contact with the oolemma. In order to improve our understanding of gamete interaction in cases of failed fertilization, we have analysed the failed fertilized oocytes from both SUZI and conventional in-vitro fertilization. The fluorochrome Hoechst 33342 (which binds specifically to DNA) was used to check for the possible presence of paternal chromatin in the unfertilized oocytes. A significantly higher $(P<0.01)$ number of microinjected oocytes showed signs of fertilization $2-3$ days after sperm microinjection compared to normally inseminated oocytes, $30 / 175(17.1 \%)$ and $2 / 79(2.5 \%)$ respectively. In addition, four out of eight couples returning for a second treatment by SUZI displayed anomalies in fertilization in both cycles. The semen characteristics of patients with or without anomalies in fertilization was not different. The irregularities observed in the fertilization process infer that certain male factor patients have intrinsic sperm anomalies lying at the sperm membrane and/or chromatin level that could lead to anomalies in the appearance of the pronuclei.

Key words: chromatin/fertilization/male infertility/micromanipulation/pronuclear formation

\section{Introduction}

Sub-zonal sperm microinjection into the perivitelline space of the oocyte allows spermatozoa, previously considered subfertile, to reach the surface of the oocyte and achieve fertilization. For example, fertilization and pregnancy were obtained in humans after injection of immobile spermatozoa under the zona pellucida (Bongso et al., 1989). Although numerous pregnancies and births have been obtained using this method ( $\mathrm{Ng}$ et al., 1988; Fishel et al., 1991; Sakkas et al., 1992), the average fertilization rate remains low, indicating that sperm dysfunction cannot be totally overcome by the microinjection technique. Moreover, significantly different fertilization rates have been reported for patients with similar semen parameters, suggesting that there are inherent male gamete defects (other than immotility, low concentration and abnormal form) which render the spermatozoa unable to fertilize the oocyte, even when placed in close proximity to the oolemma (Wolf et al., 1992).

Oocytes are considered as unfertilized when no pronuclei are seen the day after insemination. However, entry of spermatozoa into the oocyte followed by a block in the process of fertilization would result in the absence of pronuclei. In addition, possible reversible arrest or a slowing in the sequence of events leading to their formation may delay the appearance of the pronuclei. Improvements in the success of gamete micromanipulation techniques will, therefore, benefit from a better understanding of the processes involved in gamete interactions. Conforming to this idea, we have investigated whether the apparently unfertilized oocytes obtained after microinjection were indeed penetrated by spermatozoa and to which stage the fertilization process had proceeded. In this study, we have examined microinjected and normally inseminated oocytes in which no pronuclei were observed until embryo transfer, using the fluorescent dye Hoechst 33342 which can stain both spermatozoal and oocyte chromatin (Debey et al., 1989; Adenot et al., 1991).

\section{Materials and methods}

\section{Patients and treatments}

Fifty-five patients (63 cycles) undergoing infertility treatment, using IVF or sperm microinjection techniques, from January to December 1992 were selected for this study. Sperm microinjection was applied to couples ( 30 patients, 38 cycles) in whom the male partner had a very poor semen quality and/or who were unable to achieve fertilization in previous IVF cycles. Included in this group were three couples in which the male's spermatozoa were collected by epididymal aspiration.

All patients were desensitized with gonadotrophin releasing hormone agonist (GnRHa) (Decapeptyl; Ferring, Switzerland). To stimulate the follicular growth, $83 \%$ of them received pure FSH (Metrodin; Serono, Switzerland) from days 14 to 17 of the GnRHa treatment and human menopausal gonadotrophin (HMG) (Pergonal; Serono, Switzerland) from day 16 until the induction of ovulation, while $17 \%$ received HMG only. Human chorionic gonadotrophin (HCG) (Profasi; Serono, Switzerland) was administered when the diameter of at least three follicles was $>17 \mathrm{~mm}$.

\section{IVF and sperm microinjection methods}

Oocytes were collected $\sim 34 \mathrm{~h}$ after HCG injection by ultrasoundguided transvaginal follicular aspiration. They were cultured in T6 medium containing $10 \%$ maternal serum at $37^{\circ} \mathrm{C}$ in an 
atmosphere of $5 \% \mathrm{CO}_{2}$ in air. For normal IVF, the oocytes were inseminated with $\sim 50000$ motile spermatozoa obtained by either swim-up migration or mini-Percoll gradient centrifugation (Ord et al., 1990). For the sperm microinjection technique, spermatozoa were previously treated with $3 \mathrm{mM}$ pentoxifylline (Sigma Pharmaceuticals, Buchs, Switzerland), selected by mini-Percoll gradient centrifugation, incubated with $3 \mathrm{mM}$ 2-deoxyadenosine (Sigma Pharmaceuticals) and carefully washed. Following this treatment between four and eight spermatozoa were injected under the zona pellucida in the same manner as previously described by Sakkas et al. (1992).

The presence of pronuclei was recorded between 16 and $20 \mathrm{~h}$ after insemination or microinjection. The remaining oocytes were subsequently observed twice a day until the day of embryo transfer, using an Olympus IMT2 microscope fitted with Hoffman optics. The embryos were transferred into the uterus of the patients 2 or 3 days after routine IVF or microinjection.

\section{Fluorescence technique}

After embryo transfer had been performed, the non-degenerated unfertilized oocytes were fixed in $3.7 \%$ formaldehyde for $30 \mathrm{~min}$ at room temperature and washed three times in phosphate buffered saline (PBS). Oocytes were then stained in $1 \mu \mathrm{g} / \mathrm{ml}$ Hoechst 33342 (Sigma Pharmaceuticals) in PBS for $30 \mathrm{~min}$ and washed three times (Conover and Gwatkin, 1988). Each oocyte was transferred into a drop of PBS on a glass slide and covered by a coverslip edged with petroleum jelly to prevent evaporation and squashing. The stained oocytes were observed under a fluorescence microscope (Leitz Diaplan) equipped with an automatic photo system. Photographs were taken using a $40 \times$ objective and Ilford film ASA 400.

\section{Fluorescent chromatin patterns}

The observed chromatin patterns were classified as follows.

(1) Immature: no polar body, with or without an intact germinal vesicle. This category included different stages ranging from fully decondensed chromatin (germinal vesicle stage) to condensed chromosomes (metaphase I).

(2) Metaphase II: with the first polar body; the chromosomes were arranged on a metaphase plate.

(3) Dispersed chromosomes: first polar body; chromosomes were still condensed but dispersed from the metaphase plate.

(4) Decondensed chromosomes: first polar body; chromosomes were decondensed to varying degrees. They may form either one or several clumps of chromatin. In some cases a nuclear membrane surrounded the chromatin and one reconstituted nucleus (Szollozi, 1971) or several micronuclei could be seen.

(5) Parthenogenetic activation: two polar bodies; the chromosomes were still condensed (metaphase III-arrest), decondensed (telophase nucleus stage) or fully decondensed and surrounded by a nuclear membrane (pronuclear stage) (Kubiak, 1989).

(6) Sperm penetration: the chromatin of the spermatozoa was decondensed in the cytoplasm of the oocyte and the maternal chromatin was between metaphase II (one polar body) and telophase II stage (two polar bodies) or both the maternal and parternal chromatin formed pronuclei.

\section{Results}

\section{Patient characteristics}

Patients were divided in two groups according to the insemination technique used: the microinjection group, in which spermatozoa were injected into the perivitelline space, and the IVF group, in which conventional in-vitro insemination was applied.

The characteristics of the microinjection and IVF patients, whose unfertilized oocytes were labelled with Hoechst 33342 and observed by fluorescence microscopy, are summarized in Table I. The microinjection and IVF groups were composed of 30 (38 cycles) and 25 patients ( 25 cycles) respectively. The mean ages of the patients and numbers of oocytes collected in the two groups did not differ. As expected, the semen quality of the male partners was poorer in the microinjection group. Fertilization rates for conventional insemination are low in Table $I$ as patients with rates of $>75 \%$ fertilization usually had only a small number of unfertilized oocytes and were not included in the study. Table II shows the semen characteristics for patients in both groups. The main indication for the sperm microinjection technique was severe oligozoospermia, but this treatment was also applied to some

Table I. Parameters of the patient groups treated with sub-zonal sperm microinjection (SUZI) or conventional in-vitro fertilization (IVF)

\begin{tabular}{lcc}
\hline & SUZI & IVF \\
\hline No. of cycles & 38 & 25 \\
Mean age (years) & $33.0 \pm 4.3$ & $33.2 \pm 5.7$ \\
No. of oocytes collected & 335 & 271 \\
No. of oocytes/cycle & $\mathrm{a}$ & $10.8 \pm 9.4$ \\
$\begin{array}{l}\text { No. of cycles: } \\
\quad \text { with fertilization }\end{array}$ & $8.8 \pm 4.9$ & 22 \\
$\quad$ without fertilization & 34 & 3 \\
Fertilization rate (\%) & 4 & 45.4 \\
\hline
\end{tabular}

${ }^{a}$ Values are means \pm SD.

Table II. Distribution of the semen characteristics for patients treated by sub-zonal sperm microinjection (SUZI) and conventional in-vitro fertilization (IVF) according to (a) sperm concentration and motility and (b) morphology

\begin{tabular}{|c|c|c|c|c|c|c|c|c|}
\hline \multirow{3}{*}{$\begin{array}{l}\text { (a) Sperm concentration } \\
\left(\times 10^{6} / \mathrm{ml}\right)\end{array}$} & \multicolumn{8}{|c|}{ Sperm motility (\%) } \\
\hline & \multicolumn{2}{|l|}{$0-9$} & \multicolumn{2}{|c|}{$10-19$} & \multicolumn{2}{|c|}{$20-50$} & \multicolumn{2}{|l|}{$>50$} \\
\hline & SUZI & IVF & SUZI & IVF & SUZI & IVF & SUZI & IVF \\
\hline $0-5$ & 6 & 0 & 5 & 0 & 9 & 1 & 2 & 0 \\
\hline $6-10$ & 1 & 0 & 1 & 0 & 2 & 2 & 1 & 0 \\
\hline $11-20$ & 0 & 0 & 1 & 1 & 1 & 0 & 0 & 1 \\
\hline$>20$ & 0 & 0 & 0 & 0 & $5^{a}$ & 8 & 0 & 12 \\
\hline
\end{tabular}

\begin{tabular}{lrr}
\hline $\begin{array}{l}\text { (b) Morphology } \\
\text { (\% normal forms) }\end{array}$ & SUZI & TVF \\
\hline $0-20$ & & \\
$21-50$ & 22 & 4 \\
$>50$ & 9 & 12 \\
\hline
\end{tabular}

Four cycles in which spermatozoa were obtained by epididymal aspiration are not included.

apatients presenting with more than two previous IVF failures. 
patients with better semen parameters who presented previous IVF failures.

\section{Chromatin patterns}

One-hundred and seventy-five and 79 unfertilized oocytes, obtained from the microinjection and IVF patients respectively, were fixed in formaldehyde on the day of embryo transfer (69 $\pm 12 \mathrm{~h}$ after microinjection or insemination). As shown in Table III, the main difference between the microinjection and the IVF groups concerned the percentage of oocytes penetrated by spermatozoa (significantly different at $P<0.0012$ ). In the microinjection group, $17.1 \%$ of the apparently unfertilized oocytes were penetrated by spermatozoa and presented different stages of the fertilization process. Among them, seven oocytes contained decondensing sperm chromatin, while the others displayed two masses (or more for polyploid oocytes) of decondensed chromatin typical of pronuclei. Seventeen of them had undergone delayed pronuclear membrane formation as detected using Nomarski optics after fixation. The pronuclear chromatin appeared either homogeneous or formed a large ring at the periphery of the pronucleus, indicating that the pronuclei were not at the same stage of development (Debey et al., 1989). In the IVF group, only two oocytes ( $2.5 \%$ of the total) appeared to have been penetrated by spermatozoa, and both of them were polyploid and displayed homogeneous pronuclear chromatin, with no pronuclear membrane visible by bright field microscopy. The different steps of fertilization we observed with the Hoechst staining of the oocytes are illustrated in Figure 1.

Table III shows that the percentage of unfertilized oocytes which were immature was lower in the microinjection group (1.7\%) than in the IVF group (13.9\%). Although these results are significantly different $(P<0.0001)$, they can be explained by a higher rate of degeneration of the immature oocytes cultured in vitro following removal of the cumulus cells. The percentages of metaphase $\Pi$ oocytes or oocytes showing abnormalities in the chromatin pattern, i.e. dispersed or decondensed chromosomes, were similar in both groups (Table III). No difference was noted for the parthenogenetic activation of the microinjected $(9.1 \%$ ) or the conventionally inseminated $(16.5 \%)$ oocytes.

Table III. Chromatin patterns of unfertilized oocytes after sub-zonal sperm microinjection (SUZI) or conventional in-vitro fertilization (IVF)

\begin{tabular}{lll}
\hline & SUZI & NF \\
& No. oocytes (\%) & No. oocytes (\%) \\
\hline Unfertilized oocytes & 175 & 79 \\
Immature & $3(1.7)$ & $11(13.9)^{\mathrm{c}}$ \\
Metaphase II & $93(53.1)$ & $35(44.3)$ \\
Dispersed chromosomes & $10(5.7)$ & $5(6.3)$ \\
Decondensed chromosomes & $18(10.3)$ & $9(11.4)$ \\
Parthenogenetic activation & $16(9.1)$ & $13(16.5)$ \\
Sperm penetration & $30(17.1)$ & $2(2.5)^{\mathrm{d}}$ \\
decondensing spermatozoon & 7 & 0 \\
pronuclei & $23^{\mathrm{a}}$ & $2^{\mathrm{b}}$ \\
Others & $5(2.9)$ & $4(5.1)$ \\
\hline
\end{tabular}

${ }^{a}$ Five and ${ }^{b}$ two oocytes were polyploid.

c,dSignificantly different at $P<0.0012$ and $P<0.0001$ respectively, using $\chi^{2}$ analysis.
Some oocytes were difficult to classify according to their chromatin pattern, so they were considered as others. In the microinjection group, one very large oocyte with two polar bodies situated on opposite sides of the oocyte, and their corresponding metaphase II plates, was probably a binucleate oocyte (Edwards, 1980). Two oocytes contained, besides the maternal chromosomes, a group of condensed chromosomes which may have been prematurely-condensed sperm chromosomes (Plachot and Crozet, 1992). The remaining two were embryos. One of them was first considered to be a multi-fragmented oocyte, but the fluorescence analysis revealed a 3-cell embryo containing one nucleus in each fragment. The other one was an embryo with two unequal blastomeres, one with a nucleus and one with chromosomes arranged on a metaphase plate. In the IVF group, two oocytes displayed premature sperm chromosome condensation. One oocyte contained no DNA and another one had condensed chromosomes together with clumps of decondensed chromatin.

\section{Comparison of patients with and without delayed fertilization}

As is apparent from Table III, the phenomenon of delayed fertilization was observed mainly in microinjected oocytes. This observation raised the question as to whether the microinjected oocytes from every patient may be abnormally fertilized. Of the 38 microinjection treatment cycles 18 displayed oocytes with delayed fertilization. This indicates that the delay in fertilization may be related to some characteristics of the gametes of the patients. However, the quality of the gametes of the patients with or without delayed fertilization did not appear to be different. The semen characteristics (sperm concentration, motility and morphology) and fertilization rate were both similar. The mean age of the female partners, which may influence the quality of the oocytes, was also similar for the cycles with and without delayed fertilization.

\section{Chromatin patterns of patients undergoing two cycles}

In the microinjection group, eight patients underwent two treatment cycles. Among these patients, four were found to display delayed fertilization in both of their treatment cycles, two showed no delayed fertilization at all and two showed delayed fertilization in one cycle but not in the other (Table IV). The observation that the same pattern may occur twice for six out of eight patients is in favour of a relationship between some presently undefined characteristics of the gametes and the occurrence of delayed fertilization.

\section{Discussion}

Using a fluorescence technique, different chromatin patterns were observed in apparently unfertilized oocytes obtained after normal IVF or sperm microinjection. Except for the oocytes penetrated by spermatozoa, the repartition of the different patterns was not different for the IVF and the microinjection group. In some unfertilized oocytes the chromosomes were dispersed from the metaphase plate, and in others they were decondensed to varying degrees. These phenomena, which were similar for both groups, could be due to ageing of the oocytes (Szollozi, 1971; 

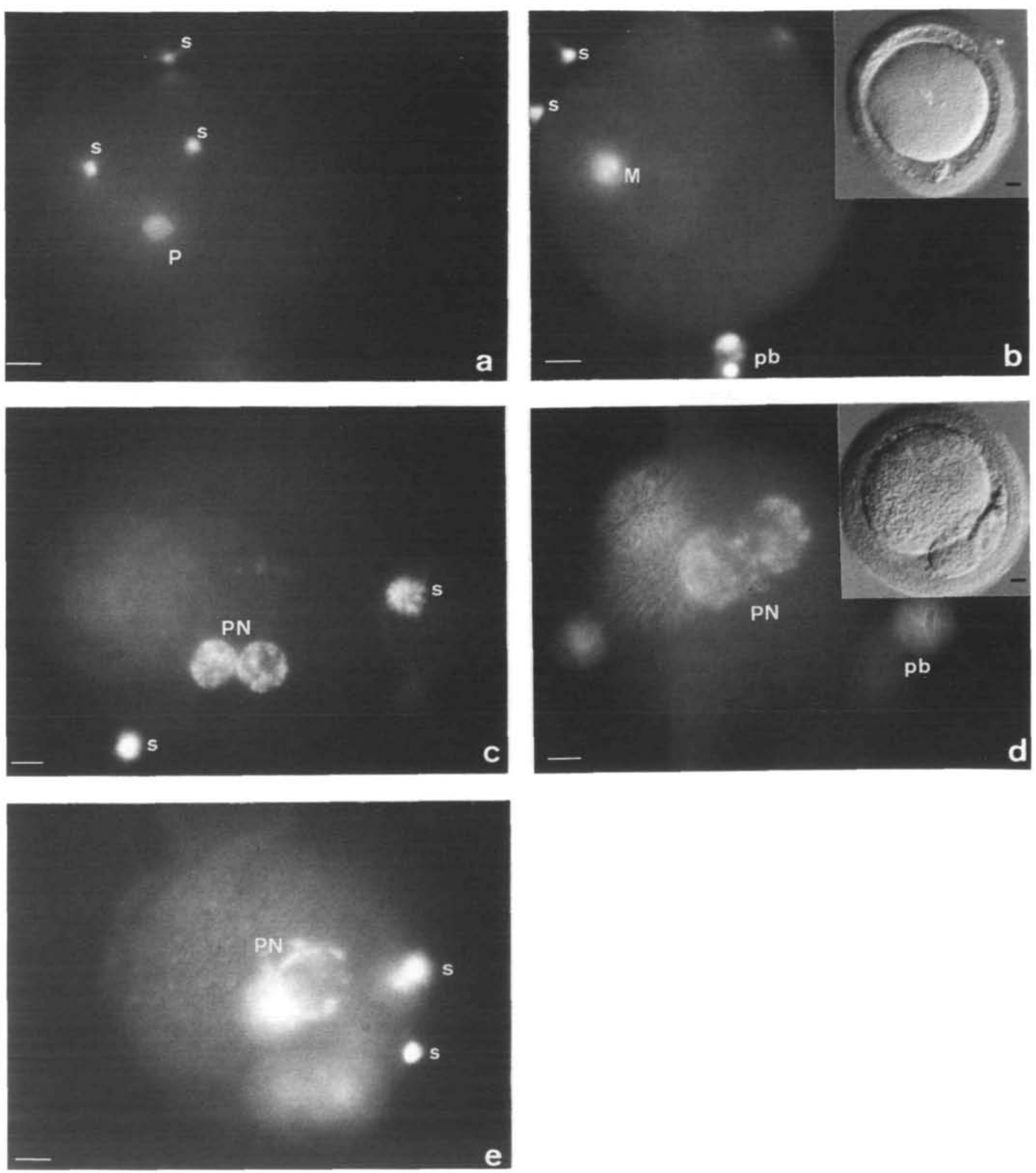

Fig. 1. Various stages of fertilization observed in assumed unfertilized microinjected oocytes after fixation and staining with Hoechst 33342 . (a) An oocyte, fixed $48 \mathrm{~h}$ after sperm microinjection, containing decondensed sperm chromatin (P). Spermatozoa (s) in the perivitelline space appeared brightly fluorescent. (b) The same oocyte but focused on the maternal chromosomes (M), which were in telophase II, and two polar bodies (pb). Insert: the same oocyte in Normarski optics. (c) An oocyte, fixed $46 \mathrm{~h}$ after microinjection, with two small pronuclei (PN). No pronuclear membrane was visible with Nomarski optics; spermatozoa (s) were present in the perivitelline space in another plane of focus. (d) Another oocyte from the same patient also had two pronuclei (PN) whose chromatin appeared homogeneous. Insert: no pronuclear membrane was visible with Nomarski optics. (e) An oocyte, fixed $62 \mathrm{~h}$ after microinjection, with two pronuclei (one in focus). The chromatin formed an incomplete ring. Bar $=10 \mu \mathrm{m}$. 
Table IV. Sperm chromatin in microinjected oocytes from patients undergoing two treatment cycles

\begin{tabular}{lll}
\hline Patient no. & $\begin{array}{l}\text { No. of oocytes labelled } \\
\text { with Hoechst } 33342\end{array}$ & $\begin{array}{l}\text { No. of oocytes } \\
\text { with sperm chromatin }\end{array}$ \\
\hline 1 & 3 & 2 \\
1 & 3 & 1 \\
2 & 4 & 2 \\
2 & 8 & 3 \\
3 & 2 & 1 \\
3 & 4 & 4 \\
4 & 6 & 3 \\
4 & 6 & 1 \\
5 & 1 & 0 \\
5 & 2 & 0 \\
6 & 13 & 0 \\
6 & 9 & 0 \\
7 & 6 & 0 \\
7 & 9 & 3 \\
8 & 6 & 1 \\
8 & 3 & 0 \\
\hline
\end{tabular}

Spermatozoa from patient 2 were aspirated from the epididymis.

Eichenlaub-Ritter et al., 1988; Pickering et al., 1988; Van Wissen et al., 1991) since they were cultured for at least 2 days prior to fixation. The rates of parthenogenetic activation were similar to those found in previous studies (Pickering et al., 1988; Balakier and Casper, 1991) and show that the microinjection procedure does not increase the rate of parthenogenetic activation, as was reported in the mouse (Mann, 1988).

The main difference between the microinjection and IVF groups was the occurrence of fertilization when embryo cleavage was expected, i.e. $2-3$ days after insemination. The presence of decondensing sperm chromatin and pronuclear chromatin so late after the microinjection may be due to an arrest in the process of fertilization following the normal entry of the spermatozoa into the oocyte. However, since the continuous follow-up of the oocyte chromatin could not be performed, it is difficult to conclude whether the development had been arrested or not. Alternatively, the sequence of events leading to the pronuclei formation may be disturbed in such a way that the appearance of pronuclei would be delayed, leading to the delayed fertilization phenomenon.

The delayed fertilization may be due to a number of factors. Firstly, the microinjection technique itself may be responsible. The handling of the oocytes is obviously different from conventional IVF and sperm microinjection. We cannot exclude the possibility that the manipulation of the oocytes, necessary for performing microinjection, may stress the oocytes and perturb the events leading to pronuclear formation. In addition, during the microinjection technique the zona pellucida, which is the physiological inducer of the acrosome reaction (Bleil and Wassarman, 1983; Cross et al., 1988), is bypassed and the acrosome reaction is expected to occur spontaneously during the preparation of spermatozoa (Mortimer et al., 1989). Sathananthan et al. (1989) have reported that many spermatozoa were still acrosome-intact when injected under the zona pellucida but were able to complete their acrosome reaction in the perivitelline space. Consequently the entry of the spermatozoon into the oocyte could be delayed. Alternatively, spontaneously acrosome-reacted spermatozoa may not be able to interact in a proper manner with the plasma membrane of the oocyte. However, the observation that only 18 of 38 microinjection cycles displayed delayed fertilization questions the involvement of the microinjection technique in that phenomenon.

More probable is that the gametes themselves have some characteristics which contribute to delayed fertilization. Indeed, from the eight patients who came for a second treatment cycle, six presented the same results: four had oocytes with delayed fertilization in the two consecutive treatment cycles, while two presented no delayed fertilization in either cycle. This observation is more in favour of a problem relating to one or both of the gametes. As oocytes were obtained from women whose average age and hormonal stimulation were similar, differences observed in the two groups concerning delayed fertilization cannot be easily attributed to the quality of the oocytes. In contrast, the semen quality of the male partner was the main difference between the microinjection and IVF groups, and a role of the fertilizing spermatozoa in delayed fertilization could therefore be hypothesized. Interestingly, the parameters responsible for the delayed fertilization could not be attributed to motility, concentration or morphology of the spermatozoa, which indicates that the problem is more intrinsic to the spermatozoa.

Attributing the cause of delayed fertilization to the spermatozoa indicates that the problem could lie either in a delay of the spermatozoon to fuse with the oolemma or a delay in decondensation in the ooplasm. A number of proposals could be put forward to associate the phenomenon of delayed fertilization with intrinsic sperm anomalies. Spermatozoa from certain subfertile men may have defects in their surface proteins and/or in their chromatin organization, leading to abnormal fertilization.

For example, the fertilizing spermatozoon is known to trigger oocyte activation (Nuccitelli, 1991) as a prelude to the event of pronuclear formation. As has been previously suggested (Miyazaki, 1990; Williams et al., 1992), the fertilizing spermatozoon may activate the oocyte by binding to a G-protein coupled receptor which is located in the plasma membrane of the oocyte. The persistent activation of the G-protein would induce the production of the second messenger, inositol trisphosphate, which in turn would cause the repeated calcium rises involved in oocyte activation (Miyazaki, 1990). A decrease of the amplitude and frequency of calcium oscillation was shown to slow down the time course of pronuclear formation in the mouse oocyte (Vitullo and Ozil, 1992). According to that model, the spermatozoon plasma membrane must contain a ligand able to bind to the putative oocyte receptor. The $\mathrm{PH}-30$ protein, isolated from the guinea-pig spermatozoon plasma membrane, appears to have both fusion and binding properties (Blobel et al., 1992) and may be considered as a potential oocyte receptor ligand. Alterations to the ligand molecules in the plasma membrane of the spermatozoon could prevent interaction between the gamete membranes, leading to a delay or prevention of binding, fusion and/or egg activation. A previous study has shown that the proteolytic processing of the guinea-pig protein $\mathrm{PH}-30$, occurring during transit of the spermatozoa in the epididymis, is a necessary step for these cells to become fertile (Blobel et al., 1990). In light of this, 
the repeated delayed fertilization observed for the patient whose spermatozoa was aspirated from the epididymis indicates that, in some patients, spermatozoa recovered from the epididymis could bear immature surface proteins limiting the chance of fertilization.

Recent observations also suggest that there may be a relationship between the organization of the spermatozoal chromatin and the success of fertilization. Spermatozoa with abnormal nuclear chromatin organization are more frequent in infertile than in fertile men (Jager, 1990; Foresta et al., 1992; Lipitz et al., 1992; Bianchi et al., unpublished results) and may lead to a reduced capacity to fertilize an oocyte in vitro (Liu and Baker, 1992). In particular, it has been reported that human male infertility may be associated with a decrease of protamine P2 content in spermatozoal chromatin (Belokopytova et al., 1993). Furthermore, as has been suggested by Griveau et al. (1992), the decondensation of the spermatozoal chromatin, which precedes the formation of the male pronucleus in the oocyte, may be hampered when the chromatin organization is abnormal. These authors found that asthenozoospermic men displayed a high percentage of spermatozoa with nuclear abnormality and, using cytoplasmic extracts from unfertilized Xenopus laevis oocytes to induce decondensation, demonstrated that their sperm chromatin decondensed slowly and partially compared to normozoospermic men. Following these observations, we may hypothesize that, although spermatozoal entry into the oocyte would be normal after microinjection, the rate of decondensation of the spermatozoal chromatin in ovo could be decreased and, consequently, the appearance of the male pronucleus would be delayed. Since we have not observed any asynchrony in the formation of male and female pronuclei, the possible slow decondensation of the male chromatin may occur while the female chromosomes are prevented from progressing to anaphase $\Pi$, to decondense and to transform into a pronucleus. This hypothesis would imply that the fertilizing spermatozoon, of certain male factor patients treated by microinjection, interferes in regulatory mechanisms controlling events leading to pronuclear formation, limiting the chances of achieving fertilization.

In our analysis of apparently unfertilized oocytes, we attributed the phenomenon of delayed fertilization to a problem concerning the patients undergoing microinjection. We have hypothesized that certain male factor patients possess intrinsic anomalies in their spermatozoa (protein surface and/or chromatin organization abnormalities) that cannot be characterized by the semen parameters of spermatozoa number, motility and morphology. The successful treatment of these patients by micromanipulation techniques would, therefore, benefit by a greater understanding of these anomalies and their resultant effect on fertilization.

\section{Acknowledgements}

We thank the technicians and clinicians involved in the routine IVF work and Dr S.Schorderet-Slatkine for use of the fluorescence microscope.

\section{References}

Adenot,P.G., Szollosi,M.S., Geze,M., Renard,J.P. and Debey,P. (1991) Dynamics of paternal chromatin changes in live one-cell mouse embryo after natural fertilization. Mol. Reprod. Dev., 28, 23-34.
Balakier,H. and Casper,R.F. (1991) A morphological study of unfertilized oocytes and abnormal embryos in human in vitro fertilization. J. In Vitro Fertil. Embryo Transfer, 8, 73-79.

Belokopytova,I.A., Kostyleva,E.I., Tomilin,A.N. and Vorob'ev,V.I. (1993) Human male infertility may be due to a decrease of the protamine 22 content of sperm chromatin. Mol. Reprod. Dev., 34, 53-57.

Bleil,J.D. and Wassarman,P.M. (1983) Sperm-egg interactions in the mouse: sequence of events and induction of the acrosome reaction by a zona pellucida glycoprotein. Dev. Biol., 95, 317-324.

Blobel,C.P., Myles,D.G., Primakoff,P. and White,J.M. (1990) Proteolytic processing of a protein involved in sperm-egg fusion correlates with acquisition of fertilization competence. J. Cell Biol., 111, 69-78.

Blobel,C.P., Wolfsberg,T.G., Turck,C.W., Myles,D.G., Primakoff,P. and White,J.M. (1992) A potential fusion peptide and an integrin ligand domain in a protein active in sperm-egg fusion. Nature, 356, $248-252$.

Bongso,T.A., Sathananthan,S.A.H., Wong,P.C., Ratnam,S.S., $\mathrm{Ng}$, S.C., Anandakumar,C. and Ganatra,S. (1989) Human fertilization by micro-injection of immotile spermatozoa. Hum. Reprod., 4, $175-179$.

Conover,J.C. and Gwatkin,R.B.L. (1988) Pre-loading of mouse oocytes with DNA-specific fluorochrome (Hoechst 33342) permits rapid detection of sperm-oocyte fusion. J. Reprod. Fertil., 82, 681-690.

Cross,N.L., Morales,P., Overstreet,J.W. and Hanson,F.W. (1988) Induction of acrosome reactions by human zona pellucida. Biol. Reprod., 38, 235-244.

Debey,P., Renard,J.P., Coppey-Moisan,M., Monnot,I. and Geze,M. (1989) Dynamics of chromatin changes in live one-cell mouse embryos: a continuous follow-up by fluoresence microscopy. Exp. Cell Res., 183, 413-433.

Edwards,R.G. (1980) The adult ovary. In Edwards,R.G. (ed.), Conception in the Human Female. Academic Press, London, p. 271.

Eichenlaub-Ritter,U., Stahl,A. and Luciani,J.M. (1988) The microtubular cytoskeleton and chromosomes of unfertilized human oocytes aged in vitro. Hum. Genet., 80, 259-264.

Fishel,S., Antinori,S., Jackson,P., Johnson,J. and Rinaldi,L. (1991) Presentation of six pregnancies established by sub-zonal insemination (SUZI). Hum. Reprod., 6, 124-150.

Foresta,C., Zorzi,M., Rossato,M. and Varotto,A. (1992) Sperm nuclear instability and staining with aniline blue: abnormal persistence of histones in spermatozoa in infertile men. Int. J. Androl., 15, 330-337.

Griveau,J.F., Charbonneau,M., Blanchard,Y., Lescoat,D. and Le Lannou,D. (1992) Decondensation of human sperm nuclei and HP1 protamine degradation from normospermia and asthenospermia in Xenopus egg extracts. Archs Androl., 29, 127-136.

Jager,S. (1990) Sperm nuclear stability and male infertility. Archs Androl., 25, 253-259.

Kubiak,J.Z. (1989) Mouse oocytes gradually develop the capacity for activation during the metaphase II arrest. Dev. Biol., 136, 537-545.

Lipitz,S., Bartoov,B., Rajuan,C., Reichart,M., Kedem,P., Mashiach,S. and Dor,J. (1992) Sperm head ultramorphology and chromatin stability of males with unexplained infertility who fail to fertilize normal human ova in vitro. Andrologia, 24, 261-269.

Liu,D.Y. and Baker,H.W.G. (1992) Sperm nuclear chromatin normality: relationship with sperm morphology sperm - zona pellucida binding, and fertilization rates in vitro. Fertil. Steril., 58, 1178-1184.

Mann,J.R. (1988) Full term development of mouse eggs fertilized by a spermatozoon microinjected under the zona pellucida. Biol. Reprod., 38, $1077-1083$.

Miyazaki,S. (1990) Cell signalling at fertilization of hamster eggs. J. Reprod. Fertil., 42 (Suppl.), 163-175.

Mortimer,D., Curtis,E.F., Camenzind,A.R. and Tanaka,S. (1989) The spontaneous acrosome reaction of human spermatozoa incubated in vitro. Hum. Reprod., 4, 57-62. 
Ng,S.C., Bongso,A., Ratnam,S.S., Sathananthan,H., Chan,C.L.K., Wong,P.C., Hagglund,L., Anandakumar,C., Wong,Y.C. and Goh,V.H.H. (1988) Pregnancy after transfer of multiple sperm under the zona. Lancet, ii, 790.

Nuccitelli,R. (1991) How do sperm activate eggs? Curr. Top. Dev. Biol., 25, $1-16$

Ord,T., Patrizio,P., Marello,E., Balmaceda,J.P. and Asch,R.H. (1990) Mini-Percoll: a new method of semen preparation for IVF in severe male factor infertility. Hum. Reprod., 5, 987-989.

Pickering,S.J., Johnson,M.H., Braude,P.R. and Houliston,E. (1988) Cytoskeletal organization in fresh, aged and spontaneously activated human oocytes. Hum. Reprod., 3, 978-989.

Plachot,M. and Crozet,N. (1992) Fertilization abnormalities in human in-vitro fertilization. Hum. Reprod., 7, 89-94.

Sakkas,D., Lacham,O., Gianaroli,L. and Trounson,A.O. (1992) Subzonal sperm microinjection in cases of severe male factor infertility and repeated in vitro fertilization failure. Fertil. Steril., 57, $1279-1288$.

Sathananthan,A.H., Ng,S.C., Trounson,A., Bongso,A., Laws-King,A. and Ratnam,S.S. (1989) Human micro-insemination by injection of single or multiple sperm: ultrastructure. Hum. Reprod. , 4, 574-583.

Szollozi,D. (1971) Morphological changes in mouse eggs due to aging in the fallopian tube. Am. J. Anat., 130, 209-226.

Van Wissen,B., Bomsel-Helmreich,O., Debey,P., Eisenberg,C., Vautier,D. and Pennehouat,G. (1991) Fertilization and ageing process in non-divided human oocytes after GnRHa treatment: an analysis of individual oocytes. Hum. Reprod., 6, 879-884.

Vitullo,A.D. and Ozil,J.P. (1992) Repetitive calcium stimuli drive meiotic resumption and pronuclear development during mouse oocyte activation. Dev. Biol., 151, 128-136.

Williams,C.J., Schultz,R.M. and Kopf,G.S. (1992) Role of G proteins in mouse egg activation: stimulatory effects of acetylcholine on the $\mathrm{ZP2}$ to $\mathrm{ZP2} f$ conversion and pronuclear formation in eggs expressing a functional $\mathrm{ml}$ muscarinic receptor. Dev. Biol., 151, 288-296.

Wolf,J.P., Ducot,B., Kunstmann,J.M., Frydman,R. and Jouannet,P. (1992) Influence of sperm parameters on outcome of subzonal insemination in the case of previous IVF failure. Hum. Reprod., 7, $1407-1413$.

Received on May 21, 1993; accepted on July 28, 1993 